\title{
Long-Term Memory in Amnesia: Cued Recall, Recognition Memory, and Confidence Ratings
}

\author{
Arthur P. Shimamura and Larry R. Squire \\ Veterans Administration Medical Center, San Diego, California and Department of Psychiatry \\ University of California, San Diego
}

\begin{abstract}
We explored what kind of information is acquired when amnesic patients are able to exhibit significant retention on tests of cued recall and recognition memory. Amnesic patients and control subjects attempted to learn sets of sentences. Memory for the last word in each sentence was tested after $1 \mathrm{hr}$ in the case of the amnesic patients, or after 1 to 2 wceks in the case of (delayed) control subjects. Amnesic patients and (delayed) control subjects performed at similar levels on tests of cued recall and recognition memory. Amnesic patients were just as confident of their correct answers as were control subjects. Moreover, amnesic patients were no more disadvantaged than control subjects when they were cued indirectly by presenting paraphrases of the original sentences. These findings demonstrate that the residual knowledge retained by amnesic patients can be as flexible, as accessible to indirect cues, and as available to awareness as the knowledge retained by (delayed) control subjects.
\end{abstract}

Many questions about the structure and organization of human memory can be usefully addressed by studying the amnesic disorders associated with brain injury or disease. One important feature of amnesia is that it is selective and does not extend to all kinds of learning and memory (for reviews see Schacter, 1987; Shimamura, in press-a; Squire, 1987; Weiskrantz, 1987). For example, amnesic patients can acquire and retain perceptuomotor and cognitive skills (Brooks \& Baddeley, 1976; Cohen \& Squire, 1980; Corkin, 1968), despite severe impairment in the ability to acquire new facts and episodes. They also exhibit normal priming effects (for a review, see Shimamura, 1986). These observations show that the brain structures damaged in amnesia, including the hippocampus (Zola-Morgan, Squire, \& Amaral, 1986), are essential for only certain kinds of memory. We have used the term declarative memory (explicit memory for facts and episodes) to describe the kind of memory that is affected in amnesia.

An underappreciated fact about amnesia is that even among severely disabled patients, considerable differences can occur in the severity of memory impairment. Many patients are capable of some degree of residual learning ability and retention, even when they are assessed with tests of explicit memory. For example, when free-recall performance fails altogether, some residual information is frequently detectable with cued recall or recognition memory tasks. Moreover, extended exposure or repetition of to-be-learned material can often improve performance and lead to enduring memory.

This research was supported by the Medical Research Service of the Veterans Administration, by National Institute of Mental Health Grant MH24600, and by the Office of Naval Research. We thank Joyce Zouzounis, Kim Rivero-Frink, and Loni Shutler for research assistance and Arthur Glenberg, Elizabeth Glisky, Andrew Mayes, Henry Roediger III, and two anonymous reviewers for helpful comments on earlier drafts.

Correspondence concerning this article should be addressed to Arthur P. Shimamura, Veterans Administration Medical Center (V116), 3350 La Jolla Village Drive, San Diego, California 92161.
One such demonstration of residual learning ability was that the severely amnesic patient H.M. was able to perform well (87.5\% correct) on a 10 -min yes-no recognition memory test in which 40 pictures were presented during study for $15 \mathrm{~s}$ each (Huppert \& Piercy, 1979). H.M., as well as other amnesic patients, can also benefit from repetition by gradually improving performance during training (Kovner, Mattis, \& Goldmeier, 1983; Milner, Corkin, \& Teuber, 1968).

The ability of even severely amnesic patients to accomplish significant learning of factual and episodic information raises questions about the nature of the residual learning ability. One possibility is that such information is similar to weak normal memory and that the knowledge acquired by amnesic patients is represented in much the same way that it is represented in normal subjects. Another possibility is that information acquired by amnesic patients is qualitatively different from the information acquired by normal subjects (for further discussion of this issue, see Meudell \& Mayes, 1982; Shimamura, in press-b). Recent work by Glisky, Schacter, and Tulving (1986a, 1986b) was consistent with this latter idea that the declarative memory successfully acquired in amnesia is qualitatively different from normal memory. They found that the knowledge retained by amnesic patients during training was relatively inflexible, hyperspecific, and narrowly available to recall. For example, patients seemed able to recall what they had learned only when information was requested by using exactly the same words that had been used during training.

The present study explored what kind of information has been acquired when amnesic patients are able to exhibit significant retention on tests of declarative (explicit) memory. In two experiments, amnesic patients attempted to learn a set of sentences. We then asked whether the knowledge acquired by the patients about these sentences was as flexible and accessible as the knowledge acquired by normal subjects. Subjects were given cued recall tests that used either the exact wording encountered during learning or paraphrases of the 
original wording that cued the learned material only indirectly. We also tested recognition memory for the learned material. If the knowledge acquired by amnesic patients is inflexible, then patients might be disproportionately impaired, in relation to control subjects, when recall is tested indirectly. Finally, we obtained confidence ratings from all subjects to determine whether patients have as much awareness as do normal subjects about the correctness or incorrectness of their responses.

\section{Experiment 1}

\section{Method}

\section{Subjects}

Amnesic patients. Ten amnesic patients were tested: 6 with alcoholic Korsakoff's syndrome, 2 with amnesia due to an anoxic episode, 1 with amnesia due to an ischemic episode, and Case N.A. The patients with Korsakoff's syndrome consisted of 4 men and 2 women living in supervised facilities in San Diego County. The remaining 4 patients were men. Of the 2 patients with amnesia due to an anoxic episode, 1 became amnesic in 1976 after a cardiac arrest (Case A.B.), and the other became amnesic in 1984 when respiratory arrest occurred during an epileptic seizure (Case L.M.). The patient with amnesia due to ischemia became amnesic in 1983 after a period of hypotension that occurred during major surgery (Case G.D.). Case N.A. has been severely amnesic for verbal material since 1960, when he sustained a stab wound to the brain with a miniature fencing foil (Kaushall, Zetin, \& Squire, 1981; Teuber, Milner, \& Vaughan, 1968). These patients have been studied for several years (Shimamura \& Squire, 1984; Squire \& Shimamura, 1986). In the present study, the results obtained for patients with Korsakoff's syndrome were identical to the results for the other amnesic patients. Accordingly, we present the patients as a single group.

The 10 amnesic patients averaged 50.7 years of age and 13.1 years of education. They had an average Wechsler Adult Intelligence ScaleRevised (WAIS-R) IQ score of 101.6. On the Wechsler Memory Scale-Revised (WMS-R), they scored 95.0 on Attention and Concentration, 73.4 on Verbal Memory, 76.3 on Visual Memory, 68.6 on General Memory, and 58.0 on Delayed Memory. The WAIS-R and WMS-R are standardized with a mean of 100 and standard deviation of 15. Thus, these patients exhibited normal WAIS-R scores and normal Attention and Concentration index scores on the WMS-R; yet they were 2 standard deviations below average on the General Memory and Delayed Memory indices.

Free recall of a short prose passage was 5.3 segments for immediate recall and 0 segments for delayed $(12 \mathrm{~min})$ recall $(21$ segments total). Average scores for copy and delayed recall $(12 \mathrm{~min})$ of a complex diagram (Rey-Osterreith figure) were 28.9 and 5.7 segments, respectively (36 segments total). Paired-associate memory of 10 unrelated noun-noun pairs on each of three successive trials was $0.7,0.4$, and 1.5. Also, on five successive study/test trials (Rey Auditory Verbal Learning Test), these patients averaged 4.1, 4.8, 5.5, 5.3, and 5.1 for free recall of 15 nouns; they averaged $21.9,24.8,25.0,26.0$, and 27.0 correct for yes-no recognition of 15 old nouns and 15 new nouns.

Neuropsychological screening and independent neurological examination indicated that memory impairment was the only remarkable deficit of higher cortical function. The amnesic patients averaged 132.5 points out of a possible 144 points on the Dementia Rating scale (Mattis, 1976), losing most of their points on the memory subportion of the test. All patients could draw a cube and a house in perspective, and none had aphasia or apraxia. Additional neuropsy- chological data for these 10 patients, as well as the data just summarized, can be found in Squire and Shimamura (1986).

Alcoholic control subjects. We tested 14 alcoholic control subjects ( 8 men and 6 women) who were current or former participants in San Diego County alcohol treatment programs. They had an average drinking history of 18.8 years but had abstained from alcohol for an average of 1.2 years prior to participating in the experiment. These subjects averaged 51.0 years of age, averaged 12.9 years of education, and obtained an average WAIS-R subtest score of 19.7 for Information ( 19.7 for the amnesic patients) and 48.3 for Vocabulary ( 48.4 for the amnesic patients). Their scores for immediate and delayed recall (12 min) of a short prose passage averaged 7.6 and 6.0 segments, respectively. We assigned these alcoholic subjects to two groups. The first group (6 subjects) was tested after a 1-hr retention interval just as the amnesic patients werc. The second group ( 8 subjects) was tested after a 1- or 2-week retention interval (average interval was 1.5 weeks) to match their level of performance to that of the amnesic patients.

Healthy control subjects. We tested 20 healthy control subjects (11 men and 9 women) who were employees or volunteers at the San Diego Veterans Administration Medical Center. They averaged 46.6 years of age and 15.1 years of education and obtained an average WAIS-R subtest score of 21.8 for Information (19.7 for the amnesic patients) and 53.5 for Vocabulary (48.4 for the amnesic patients). Their scores for immediate and delayed recall $(12 \mathrm{~min})$ of a short prose passage averaged 8.0 and 5.9 segments, respectively. We assigned these healthy control subjects to two groups. The first group ( 8 subjects) was tested after a 1 -hr retention interval just as the amnesic patients were. The second group (12 subjects) was tested after a 1- or 2-week retention interval (average interval was 1.7 weeks).

\section{Materials}

Twenty sentences were constructed so that the last word in each sentence was a noun that could be used as a test word (e.g., "At the fair, Sarah lost her keys"; "The newlyweds went to the furniture store to look at the bookcase'). Each test word was randomly chosen from a set of four likely possibilities. (The other three words were used as distractors for a recognition test.) Sentences were printed individually on index cards. Recall was tested in two ways. In the direct recall condition, subjects were shown previously presented sentences with the test word omitted (e.g., "At the fair, Sarah lost her Subjects were asked explicitly to recall the word that was previously presented. In the indirect recall condition, subjects were also asked explicitly to recall the word that was presented. However, in this case subjects were asked questions that cued the test word somewhat indirectly (e.g., What did she lose at the fair? What did the couple inspect at the furniture store?). For each question, one or two content words used in the original sentence were replaced with a related word. Finally, a four-alternative recognition memory test was used in both the direct and indirect conditions (e.g., keys, bracelet, purse, sweater; bookcase, desk, loveseat, table).

\section{Procedure}

Subjects were shown 20 sentences on 20 cards and asked to read each sentence aloud and to try to remember each one. Presentation rate was self-paced, according to the time it took subjects to read each sentence (approximately $4 \mathrm{~s}$ per sentence). After all 20 sentences were presented, the cards were shuffled, and the sentences were presented a second time. Following a 1-hr retention interval, recall for 10 of the sentences was tested in the direct recall condition, and recall for the other 10 sentences was tested in the indirect recall condition. Sentences were counterbalanced across direct and indirect conditions. For half of the subjects, testing in the direct condition preceded testing in the indirect condition, and for the other half the test order 
was reversed. Both test conditions were presented orally, and subjects responded verbally. Subjects werc cncouraged to guess if they were unsure of the correct answer, but no feedback was provided to subjects about their answers. Subjects were also asked to rate on a 5-point scale their confidence that the response was correct $(1=$ low and $5=$ high). A card indicating the scale was placed in front of the subjects throughout testing.

After the recall test a four-alternative forced-choice recognition test was given. Half of the 10 sentences used in each of the two recall conditions were assessed by using the direct testing method (the original sentence with the final word omitted and four possible choices), and the other half were assessed by using the indirect testing method (a question with one or two content words altered and four possible answers). The recognition test was written on a single form, and subjects indicated their choices verbally. Two groups (alcoholic controls and healthy controls) were tested after a 1- to 2-week delay in order to match their recall performance in the direct condition to that of the amnesic patients. Other than the difference in retention interval, these two delayed control groups were tested in the same manner as were the groups tested after a 1-hr delay.

To assess the baseline tendency to use test words to complete these sentences, we gave the test items to a new group of 12 healthy control subjects ( 7 men, with a mean age of 53.7 years and a mean education of 14.5 years) without prior presentation of the sentences. For each subject, 10 words were cued by asking subjects to read sentences with the final word missing (direct cue), and 10 words were cued by questions (indirect cue). In each case, subjects were asked to say the first word that came to mind that could plausibly complete the sentence. The order in which the cue types (direct and indirect) were presented was counterbalanced across subjects.

\section{Results and Discussion}

Figure 1 displays direct and indirect recall performance for healthy control subjects, alcoholic subjects, amnesic patients tested after a 1-hr delay, and delayed healthy control subjects

\section{RECALL}

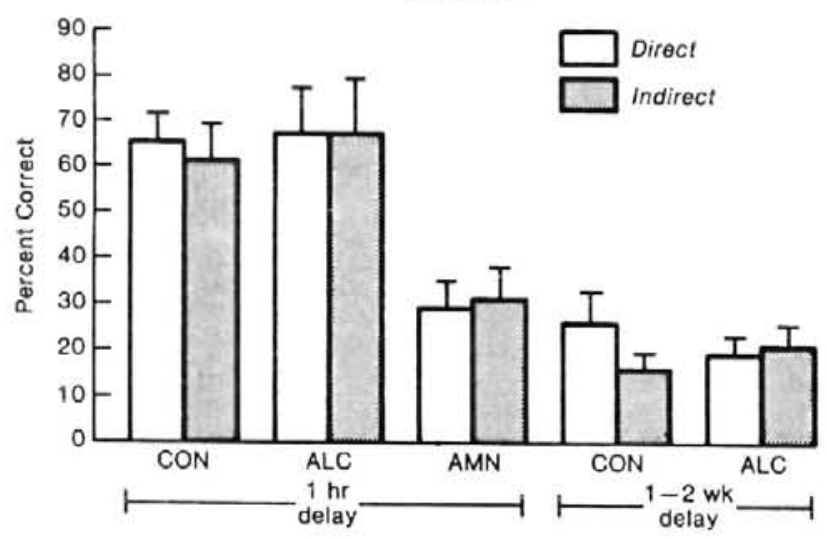

Figure 1. Recall of test words by healthy control subjects (CON), alcoholic control subjects (ALC), and amnesic patients (AMN) tested after a 1-hr delay in Experiment 1. Two other groups of healthy control subjects and alcoholic subjects were tested after a 1- to 2 . week delay. Subjects were cued directly (open bars) by presenting verbatim the sentence in which the test word originally appeared (At the fair, Sarah lost her ), or they were cued indirectly (shaded bars) with questions in which one or two of the original content words were replaced with related words (What did she lose at the fair?). and delayed alcoholic subjects tested after 1- to 2-week delay. Recall performance by all subject groups was significantly above baseline levels (baseline was 3\% for the direct condition and $7 \%$ for the indirect condition). Subjects provided responses for about $95 \%$ of the questions. The data were submitted to a $5 \times 2$ analysis of variance (ANOVA) with group and recall condition (direct or indirect) as independent variables. There was a significant effect of group, $F(4,39)=14.6$, $p<.001, M S_{\mathrm{c}}=570$. Planned comparisons indicated that performance of healthy control subjects and alcoholic subjects were significantly better than was the performance of amnesic patients, delayed healthy controls, or delayed alcoholic subjects $(t s>2.8, p s<.01)$. The latter three groups performed similarly $(t \mathrm{~s}<0.9, p \mathrm{~s}>.2)$. There was no significant main effect of cued recall condition, $F(1,39)=1.1$, although the difference between direct and indirect conditions approached significance in the case of the delayed healthy control subjects, $t(11)=2.1, p=.06$. There was no significant interaction between group and cued recall condition, $F(1,4)=1.08$.

Figure 2 displays confidence ratings for correct and incorrect responses for both direct and indirect conditions. The data were submitted to a $5 \times 2 \times 2$ ANOVA with group, recall condition, and response accuracy as independent variables. There was a main effect of response accuracy, that is, confidence ratings were greater for correct responses than for incorrect responses, $F(1,4)=239, p<.001, M S_{\mathrm{e}}=.50$. There was also a significant Group $\times$ Response Accuracy interaction, $F(4,29)=3.2, p=.02$, which was due to the fact that healthy control subjects and alcoholic subjects tested after a 1-hr delay discriminated correct from incorrect responses better than did amnesic patients, delayed healthy controls, or delayed alcoholic controls. The latter three groups performed similarly $(t s<0.7, p s>.2)$. The only other significant finding was a main effect of recall condition, $F(1,4)=4.1, p=.05$, which indicated that, averaged across correct and incorrect responses, confidence ratings in the direct condition were greater than were ratings in the indirect condition.

Recognition memory was assessed after the cued recall test, and thus this test did not provide a pure measure of recognition memory performance. Nevertheless, recognition performance by the subject groups paralleled cued recall performance (see Table 1). All groups performed above the expected chance level (chance $=25 \%$ ). There was no effect of type of recognition cue (direct vs. indirect condition), $F(1,39)=0.3$, $p>.4, M S_{\mathrm{e}}=2.2$. Both healthy and alcoholic control subjects exhibited better recognition performance than did amnesic patients, delayed healthy control subjects, or delayed alcoholic subjects $(t \mathrm{~s}>2.3, p \mathrm{~s}<.05)$. The amnesic patients and the two delayed groups performed similarly, although the amnesic patients did exhibit slightly better recognition memory than did delayed alcoholic subjects, $t(16)=1.8, p=.09$. Finally, there was no overall Group $\times$ Cue Condition interaction, $F(4$, 39) $=0.6, p>.4, M S_{\mathrm{e}}=2.2$.

In this experiment, cued recall and recognition were affected similarly in amnesic patients and control subjects (see Table 1). For example, the differences between overall recognition memory and cued recall performance in amnesic patients, delayed alcoholic subjects, and delayed healthy control subjects were $26 \%, 17 \%$, and $28 \%$, respectively. Thus, the amnesic patients gave little indication that their cued 


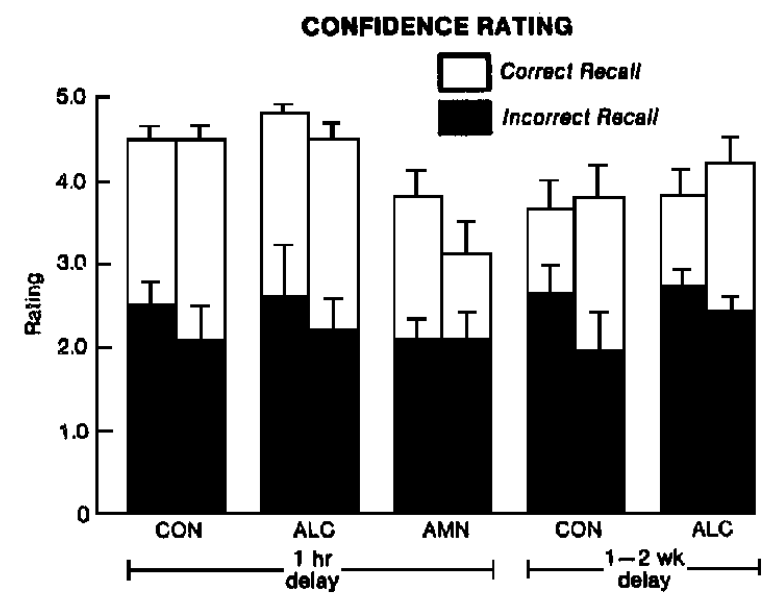

Figure 2. Confidence ratings for recall responses in Experiment 1 by healthy control subjects (CON), alcoholic control subjects (ALC), and amnesic patients (AMN) tested after a 1-hr delay. Two other groups of healthy control subjects and alcoholic subjects were tested after a 1- to 2-week delay. Bars show mean confidence ratings for correctly recalled responses, and solid regions show mean confidence ratings for incorrectly recalled responses. For each subject group the left bar shows confidence ratings for responses in the direct recall condition, and the right bar shows confidence ratings for responses in the indirect recall condition.

recall memory performance was disproportionately impaired in relation to recognition memory performance. These findings can be contrasted with findings from Hirst and colleagues (Hirst et al., 1986; Hirst, Johnson, Phelps, \& Volpe, 1988), who found that free-recall performance was disproportionately impaired in amnesic patients.

\section{Experiment 2}

In the first experiment, amnesic patients performed as well as did delayed control subjects when the target words from the original sentences were cued somewhat indirectly. In addition, the confidence ratings reported by amnesic patients for correctly recalled information (i.e., their familiarity ratings) were similar to those reported by delayed control sub-

Table 1

Experiment 1: Cued Recall and Recognition Performance (\%)

\begin{tabular}{lccccccc}
\hline & \multicolumn{3}{c}{ Cued recall } & & \multicolumn{3}{c}{ Recognition } \\
\cline { 2 - 4 } \cline { 6 - 8 } Group & Direct & Indirect & Overall & & Direct & Indirect & Overall \\
\hline CON & 65 & 61 & 63 & & 83 & 86 & 84 \\
ALC & 67 & 67 & 67 & & 87 & 78 & 82 \\
AMN & 29 & 31 & 30 & & 60 & 53 & 56 \\
CON-D & 26 & 15 & 21 & & 46 & 52 & 49 \\
ALC-D & 19 & 21 & 20 & & 35 & 40 & 37 \\
\hline
\end{tabular}

Note. $\mathrm{CON}=$ healthy control subjects, tested after a 1-hr delay; ALC = alcoholic subjects, tested after a 1-hr delay; AMN = amnesic patients, tested after a 1 -hr delay; $\mathrm{CON}-\mathrm{D}=$ healthy control subjects, tested after a 1- to 2-week delay; ALC-D = alcoholic subjects, tested after a 1- to 2-week delay. jects. These results suggest that the information acquired by amnesic patients and tested by cued recall is not markedly hyperspecific. In Experiment 2, a stronger test of this idea was achieved by wording the indirect questions so differently from the original wordings that control subjects performed more poorly in the indirect condition than in the direct condition. In addition, we gave four study/test trials to achieve greater initial learning and a higher subsequent retention score. We then asked whether amnesic patients performed similarly to (delayed) control subjects.

\section{Method}

\section{Subjects}

Amnesic patients. We tested the same patients used in Experiment 1 . They were tested at least 6 months after participating in Experiment 1 .

Alcoholic control subjects. We tested a new group of 10 alcoholic control subjects ( 7 men and 3 women). They were current or former participants in county alcoholic treatment programs, who had an average drinking history of 16.3 years but had abstained from alcohol for an average of 3.1 years prior to participating in the experiment. These subjects averaged 54.6 years of age, averaged 12.5 years of education, and obtained an average WAIS-R subtest score of 19.2 for Information (19.7 for the amnesic patients) and 46.2 for Vocabulary ( 48.4 for the amnesic patients). Their scores for immediate and delayed recall (12 $\mathrm{min}$ ) of a short prose passage averaged 7.2 and 5.4 segments, respectively.

Healthy control subjects. We also tested a new group of 10 healthy control subjects ( 9 men and 1 woman). They averaged 54.0 years of age, averaged 14.1 years of education, and obtained an average WAIS-R subtest score of 22.0 for Information (19.7 for the amnesic patients) and 49.7 for Vocabulary (48.4 for the amnesic patients). Their scores for immediate and delayed recall $(12 \mathrm{~min})$ of a short prose passage averaged 7.4 and 5.9 segments, respectively.

\section{Materials and Procedure}

We constructed 20 new sentences similar to the ones used in Experiment 1 (e.g., The cocker spaniel chased the cat into the barn; The farmer fed his goat some barley). Subjects were given four study/ test learning trials. For each learning trial, subjects first read aloud each of the sentences one at a time. Presentation rate was self-paced according to how long it took subjects to read the sentences (approximately $4 \mathrm{~s}$ per sentence). After each learning trial, subjects were read the sentences with the target word missing and were asked to recall the missing word. If the word could not be recalled, it was provided by the experimenter. The order in which the sentences were presented was different for study and test phases and was also different across study/test trials. After the four learning trials, we tested recall and recognition under both direct and indirect conditions. The amnesic patients were tested after a 1-hr delay. Only delayed control subjects (alcoholic and healthy control subjects) were tested. These two control groups were tested after a 2-week delay, at a time when their performance on the direct recall condition matched that of the amnesic patients tested after a 1-hr delay.

For the direct condition, the original sentence was presented with the target word missing, and subjects were asked explicitly to recall the word. For the indirect condition, we altered the original sentence considerably, changing all but one of the originally presented words (e.g., Where did the dog go? What did the farm animal eat?). Subjects 
were asked explicitly to answer the questions on the basis of the previously presented sentences. Recall for 10 of the sentences was tested in the direct condition, and recall for 10 sentences was tested in the indirect condition according to the same design and procedure used in Experiment 1. As in Experiment 1, confidence ratings were recorded for each recall response $(1=$ low and $5=$ high $)$, and a fouralternative forced-choice recognition test was given in both the direct and indirect conditions. Finally, baseline guessing rates were determined by giving the direct and indirect test, but without prior presentation of the sentences, to the same 12 subjects who were given the baseline test for Experiment 1.

\section{Results and Discussion}

Table 2 shows performance across the four acquisition trials. In comparison with healthy control subjects and alcoholic subjects, amnesic patients exhibited significantly poorer learning, $F(2,25)=23, p<.01, M S_{\mathrm{e}}=19.9$. Amnesic patients did exhibit relatively good learning across the four acquisition trials, whereas rather meager learning is typically observed for unrelated word lists or unrelated paired associates. The better learning in this experiment was probably due to the semantic associations that linked sentence cues with their target words (see also Shimamura \& Squire, 1987).

Figure 3 shows that the recall performance of amnesic patients after $1 \mathrm{hr}$ was similar to the recall performance of the two control groups tested after 2 weeks, $F(2,27)=0.7$. All subject groups performed significantly above baseline levels (baseline $=5 \%$ for direct condition and $1 \%$ for indirect condition). Subjects provided responses for about $90 \%$ of the questions. There was a significant effect of recall condition (direct vs. indirect), $F(1,27)=31.6, p<.001, M S_{\mathrm{c}}=1.8$. However, there was no interaction of Group $\times$ Recall Condition, $F(2,27)=0.6, p=.5, M S_{\mathrm{c}}=1.8$, indicating that the difference between the direct and indirect conditions was similar across groups (amnesics, $21 \%$; healthy controls, $24 \%$; and alcoholics, $20 \%$ ). Thus, the decremental effect of indirect cuing was no greater for amnesic patients than that for the control subjects.

Figure 4 displays confidence ratings for correct and incorrect recall responses for both direct and indirect conditions. As in Experiment 1, all groups exhibited greater confidence for correct responses than for incorrect responses, $F(1,27)=$ $202, p<.001, M S_{\mathrm{e}}=.50$, and there was no main effect of group, $F(2,27)=0.9, M S_{\mathrm{e}}=1.2$. There was, however, a significant Group $\times$ Response Accuracy interaction, $F(2,27)$ $=4.7, p=.02, M S_{\mathrm{c}}=.50$, owing to the fact that amnesic patients and control subjects reported similar confidence rat-

Table 2

Experiment 2: Learning Across Four Study/Test Trials (\%)

\begin{tabular}{lcrrr}
\hline & \multicolumn{4}{c}{ Learning trial } \\
\cline { 2 - 5 } Group & 1 & 2 & 3 & 4 \\
\hline AMN & 32 & 58 & 74 & 82 \\
CON-D & 70 & 100 & 100 & 100 \\
ALC-D & 70 & 99 & 100 & 100 \\
\hline
\end{tabular}

Note. $\mathrm{AMN}=$ amnesic patients tested after a 1 -hr delay; CON-D = delayed healthy control subjects tested after a 2-week delay; ALC-D $=$ delayed alcoholic subjects tested after a 2-week delay.

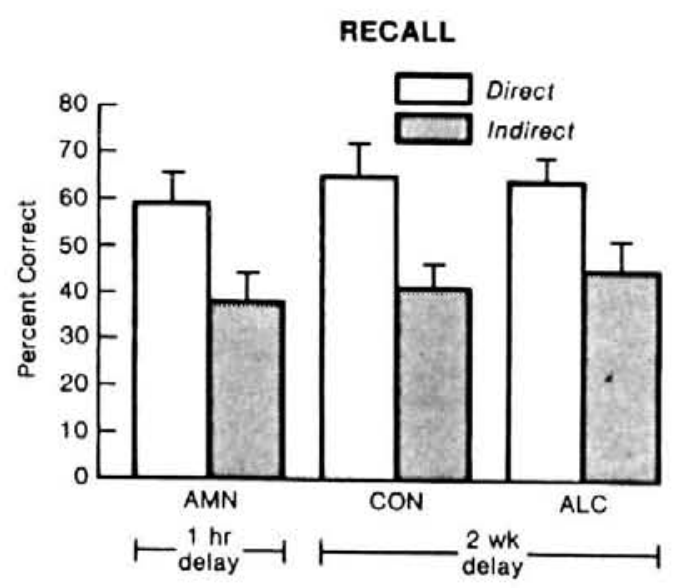

Figure 3. Recall of test words in Experiment 2 by amnesic patients (AMN) tested after a 1-hr delay and by healthy control subjects (CON) and alcoholic subjects (ALC) tested after a 2-week delay. Subjects were cued directly (open bars) by presenting verbatim the sentence in which the test word originally appearcd (The farmer fed his goats some ___ with questions in which all but one of the original content words were replaced with related words (What did the farm animal eat?).

ings for their correct responses, whereas amnesic patients reported somewhat higher ratings than did control subjects for their incorrect responses.

As in Experiment 1, recognition performance by the subject groups paralleled recall performance (see Table 3 ). All groups performed above the expected chance level (chance $=25 \%$ ). There was no difference in recognition memory performance across groups, $F(2,27)=1.7, p=.2, M S_{\mathrm{e}}=2.2$. Recognition performance in the indirect testing condition was poorer than that in the direct testing condition, $F(1,27)=6.0, p=0.2$, $M S_{\mathrm{e}}=1.0$, but there was no Group $\times$ Condition interaction, $F(2,27)=0.4, p>.4, M S_{\mathrm{e}}=.99$. Finally, as in Experiment 1 , recall and recognition were affected similarly across groups. Averaged over direct and indirect conditions, the difference between recall and recognition performance was $35 \%$ for amnesic patients, $37 \%$ for alcoholic control subjects, and $36 \%$ for delayed healthy control subjects.

\section{General Discussion}

This study explored the residual ability of amnesic patients to acquire declarative knowledge. In Experiment 1, patients read a set of sentences and then $1 \mathrm{hr}$ later were given a cue together with explicit instructions to recall the final word of each sentence. Delayed control subjects were tested at a retention interval of 1 or 2 weeks. As we had intended, the performance of the delayed control subjects matched the performance of the amnesic patients in the direct cue condition, in which target words were cued by presenting the exact wording of the original sentences. The important finding was that delayed control subjects and amnesic patients also performed the same in the indirect cue condition, in which target words were cued by presenting paraphrases of the original sentences. 


\section{CONFIDENCE RATING}

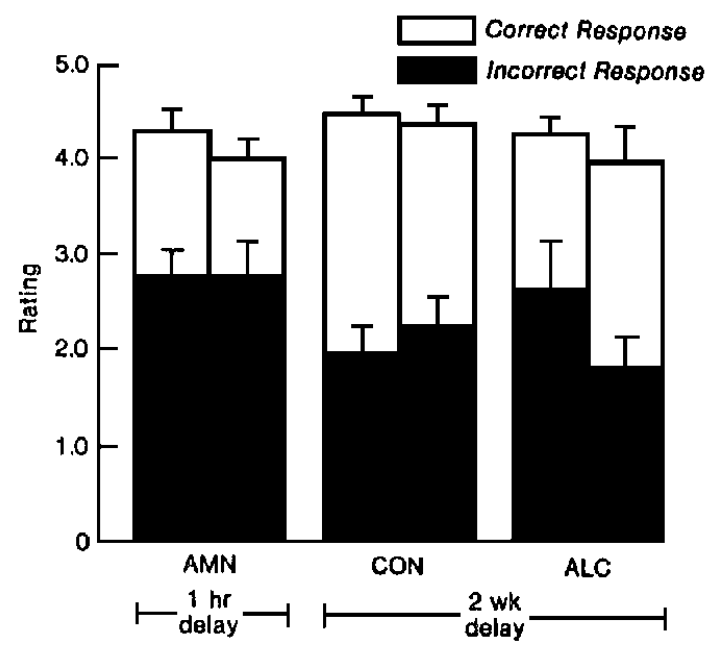

Figure 4. Confidence ratings for recall responses in Experiment 2 by amnesic patients (AMN) tested after a 1 -hr delay and by healthy control subjects (CON) and alcoholic subjects (ALC) tested after a 2week delay. Bars show mean confidence ratings for correctly recalled responses, and solid regions show mean confidence ratings for incorrectly recalled responses. For each subject group the left bar shows confidence ratings for responses in the direct recall condition, and the right bar shows confidence ratings for responses in the indirect recall condition.

In Experiment 2 we gave subjects four study/test trials in order to achieve a high level of initial learning and a high retention score. Moreover, we used paraphrases in the cued recall test that were so different from the original sentences that control subjects performed significantly more poorly in the indirect cue condition than in the direct cue condition. The results were that amnesic patients matched the delayed control subjects in the direct cue condition and then declined to the same level as did the control subjects in the indirect cue condition. Specifically, the decline in cued recall between the direct and indirect cue conditions was $21 \%$ in amnesic patients, $19 \%$ in delayed alcoholic subjects, and $24 \%$ in delayed healthy control subjects. Thus, the amnesic patients gave no suggestion that the information they retained after a 1-hr retention interval was less accessible or less flexible than the information retained by control subjects after a 1 - or 2 week retention interval.

Table 3

Experiment 2: Cued Recall and Recognition Performance (\%)

\begin{tabular}{lccccccc}
\hline & \multicolumn{3}{c}{ Cued recall } & & \multicolumn{3}{c}{ Recognition } \\
\cline { 2 - 4 } \cline { 8 - 8 } Group & Direct & Indirect & Overall & & Direct & Indirect & Overall \\
\hline AMN & 59 & 38 & 48 & & 87 & 79 & 83 \\
CON-D & 65 & 41 & 53 & & 93 & 85 & 89 \\
ALC-D & 64 & 45 & 54 & & 93 & 90 & 91 \\
\hline
\end{tabular}

Note. $\mathrm{AMN}=$ amnesic patients tested after a 1 -hr delay; $\mathrm{CON}-\mathrm{D}=$ delayed healthy control subjects tested after a 2-week delay; ALC.D $=$ delayed alcoholic subjects tested after a 2 -week delay.
We considered whether the failure to find significant differences between amnesic patients and control subjects was caused by insufficient statistical power resulting from a small sample of 10 amnesic patients. This possibility seems unlikely for several reasons. First, amnesic and control groups not only performed in a similar manner on the cued recall tests as judged by statistical measures but also produced nearly the same numerical score. Second, in Experiment 1 the amnesic patients performed slightly better (by $2 \%$ ) in the indirect cue condition than in the direct cue condition. Third, in Experiment 2 the decline in performance between direct and indirect conditions was actually greater (though only by $3 \%$ ) for delayed healthy control subjects than for amnesic patients. In these comparisons even the direction of the direct-indirect effect argues against the hypothesis that amnesic patients exhibit a disproportional deficit in the indirect cue condition. In Experiment 2 amnesic patients did exhibit a decline in the indirect cue condition that was $2 \%$ greater than the decline in alcoholic control subjects. This difference was the largest effect size we obtained in any of the comparisons.

In both experiments we also assessed confidence ratings reported by subjects during the cued recall tests. The amnesic and delayed control groups reported similar confidence ratings for the items that they recalled correctly, and both groups were able to discriminate between correct and incorrect responses. Moreover, in both experiments amnesic patients and control subjects performed similarly, even when the cued recall score was recalculated, counting only those responses for which subjects were highly confident (ratings of 4 or 5). For example, in Experiment 2 direct and indirect recall performance conditionalized on only highly confident responses was $50 \%$ and $27 \%$, respectively, for amnesic patients, $53 \%$ and $35 \%$ for healthy control subjects, and $56 \%$ and $34 \%$ for alcoholic subjects.

These findings argue against the possibility that amnesic patients were using some kind of priming ability in the cued recall test. If they were using implicit or unconscious priming ability, then many of the correct responses of amnesic patients would have come from responses given low confidence ratings. Such priming effects may operate when shorter retention intervals are used (e.g., Shimamura \& Squire, 1984), but in the present study we tested cued recall at a time (1-hr delay) when transient priming effects should have been substantially reduced. Thus, in the present study amnesic patients seemed to be aware of what they knew and of what they did not know. Others have also reported that amnesic patients can report accurate confidence ratings (Hirst et al., 1986; Hirst et al., 1988; Mayes, Meudell, \& Neary, 1980; Meudell \& Mayes, 1984).

The accurate confidence ratings given by the amnesic patients demonstrated that they were explicitly aware of their cued recall responses. Previously, we showed that patients with Korsakoff's syndrome could not make accurate feelingof-knowing judgments when they were asked to predict their performance on a subsequent recognition memory test (Shimamura \& Squire, 1986). Patients with Korsakoff's syndrome were also unreliable and inaccurate in making judgments about their own memory abilities (Squire \& Zouzounis, in press). Other amnesic patients, however, could make accurate 
feeling-of-knowing predictions as well as accurate memory self-ratings. In the present study all patients, including patients with Korsakoff's syndrome, were able to give accurate confidence ratings. Taken together, these findings suggest that familiarity or confidence judgments that follow memory responses may be easier than either predictions about performance that precede memory responses (e.g., feeling-of-knowing judgments) or ratings of memory ability made outside of any particular memory test context. Feeling-of-knowing predictions and self-ratings may require more inferential ability and more elaborate retrieval strategies than do confidence ratings. Such strategies may be impaired in patients with Korsakoff's syndrome who are known to have cortical damage, including frontal lobe damage (Shimamura, Jernigan, \& Squire, in press).

The findings for recognition memory paralleled the findings for cued recall in that the performance of amnesic patients and delayed control subjects was similar. These results were obtained even though recognition memory performance was significantly poorer with indirect cues than with direct cues (Experiment 2). Thus, as measured by both cued recall and recognition tests, the information acquired by amnesic patients was qualitatively similar to the information acquired by normal subjects. These findings can be contrasted with the findings of Hirst and colleagues (Hirst et al., 1986; Hirst et al., 1988), who reported that amnesic patients exhibited a disproportionate deficit of free recall when recognition memory was matched between amnesic patients and control subjects. Free recall may require more extensive use of retrieval strategies than does cued recall or recognition tests. Significantly, the present findings identify a boundary condition: Amnesic patients do not exhibit disproportionate deficits in cued recall tests, even when the cues are indirect and only weakly associated with the to-be-remembered information.

We attempted to test the hypothesis that residual performance by amnesic patients on tests of declarative (explicit) memory might reflect the operation of a separate, preserved memory ability. That hypothesis would be supported by demonstrating that residual memory in amnesic patients is qualitatively different from the memory of control subjects. Yet, when memory strength was matched, we found that amnesic patients were no more disadvantaged than were control subjects when recall was cued indirectly with wording that differed markedly from the wording used during original learning. Moreover, amnesic patients were just as confident of their correct and incorrect answers as were control subjects. Thus, in these two experiments the residual knowledge retained by amnesic patients, and assessed by explicit recall and recognition tests, was as flexible, as accessible to indirect cues, and as available to awareness as the knowledge retained by delayed control subjects.

In two previous studies, Glisky et al. (1986a, 1986b) suggested that through repetition, amnesic patients acquire information that is rigidly organized, hyperspecific, and inflexible. Their first study required patients to learn 30 computerrelated terms (e.g., save, loop) in response to 30 definitions. During nine sessions of training on different days, amnesic patients were impaired, but they did gradually learn the terms. Cued recall tests were then given, similar to the direct and indirect recall tests used in our two experiments. Amnesic patients performed poorly on a transfer (indirect) test, in which the 30 terms were cued with sentences that altered the wording of the original definitions. In the transfer test amnesic patients recalled $44 \%$ of the vocabulary terms learned previously, whereas control subjects recalled $63 \%$ of the previously learned terms. This measure of transfer was based on the number of terms originally learned, because the amount of original learning was different for patients and control subjects. A delayed control group would provide better evidence for poor transfer by amnesic patients. By testing normal subjects with weak or degraded memory, one can ask whether amnesic patients exhibit a normal decline or a disproportionate decline in performance on a transfer task.

There were several differences between our study and the transfer study of Glisky et al. (1986b). First, Glisky et al. (1986b) trained subjects on vocabulary words across nine study sessions scheduled on separate days, whereas we trained subjects on words on four trials scheduled on a single day. Consequently, overlearning was probably greater in the Glisky et al. (1986b) study than it was in our study. Second, Glisky et al. (1986b) asked subjects to learn rather novel vocabulary terms, whereas we asked subjects to remember words that had been embedded in common sentences. Moreover, in the Glisky et al. (1986b) study, the vocabulary terms all pertained to aspects of computer programming, whereas the sentences in our study pertained to a variety of semantic categories. Further studies that manipulate these factors would be needed to identify conditions under which inflexible, hyperspecific memory representations are established in amnesic patients.

In their second study (Glisky et al, 1986a) amnesic patients learned additional computer commands in the context of acquiring and practicing computer skills. The patients learned abnormally slowly. In addition, it was observed that, unlike control subjects, patients could not answer open-ended, general questions about what they had learned. Patients also could not answer questions that had been part of the training program when the wording of the questions was changed. Finally, patients could not demonstrate simple programming skills by applying their skills to the task of writing a new computer program. In this sense the information acquired by the amnesic patients was hyperspecific, inflexible, and accessible only in circumstances that closely resembled the conditions of original training.

The results of Glisky et al. (1986a, 1986b) could reflect the fact that amnesic patients gradually acquired some computer (procedural) skills, but they did not acquire as much declarative knowledge in conjunction with these skills as did normal subjects. That is, hyperspecificity may occur when the memory acquired by amnesic patients depends more on procedural memory (or priming) than does the memory acquired by normal individuals. Perhaps extensive repetition or overlearning produces an encapsulated or inflexible procedural memory representation. Some skills, especially in the initial stages of learning, depend not just on procedural learning but also on the mediation of explicit, declarative knowledge (Anderson, 1982). To the extent that skill learning depends on both declarative and procedural knowledge, amnesic patients should acquire some knowledge, but they should 
acquire it abnormally slowly, and their knowledge should be inflexible to some degree. Declarative knowledge is considered to be flexible, broadly available, and consciously accessible, whereas procedural knowledge is domain specific and available only to the response systems engaged during learning (Squire, 1987). Studies concerning the qualitative aspects of complex skill learning in both amnesic patients and normal subjects may illuminate the separate contributions of procedural and declarative memory.

In the present study we explored the residual memory that was observed in amnesic patients and that was assessed by explicit cued recall and recognition memory tests. We found that cued recall performance of amnesic patients was similar to the performance of delayed control subjects, even when cues were rather indirectly related to target words. Moreover, amnesic patients could discriminate correct responses from incorrect ones in an explicit manner, as is indicated by valid confidence ratings. These results suggest that in these tests of cued recall and recognition, the memory exhibited by amnesic patients was dependent on residual declarative memory, similar to that available in normal weak memory.

\section{References}

Anderson, J. R. (1982). Acquisition of cognitive skill. Psychological Review, 89, 369-406.

Brooks, D. N., \& Baddeley, A. D. (1976). What can amnesic patients learn? Neuropsychologia, 14, 111-122.

Cohen, N., \& Squire, L. R. (1980). Preserved learning and retention of pattern analyzing skill in amnesia: Dissociation of knowing how and knowing that. Science, 210, 207-209.

Corkin, S. (1968). Acquisition of motor skill after bilateral medial temporal excision. Neuropsychologia, 6, 255-265.

Glisky, E. L., Schacter, D. L., \& Tulving, E. (1986a). Computer learning by memory-impaired patients: Acquisition and retention of complex knowledge. Neuropsychologia, 24, 313-328.

Glisky, E. L., Schacter, D. L., \& Tulving, E. (1986b). Learning and retention of computer-related vocabulary in memory-impaired patients: Method of vanishing cues. Journal of Clinical and Experimental Neuropsychology, 8, 292-312.

Hirst, W., Johnson, M. K., Kim, J. K., Phelps, E. A., Risse, G., \& Volpe, B. T. (1986). Recognition and recall in amnesics. Journal of Experimental Psychology: Learning, Memory, and Cognition, $12,445-451$.

Hirst, W., Johnson, M. K., Phelps, E. A., \& Volpe, B. T. (1988). More on recognition and recall with amnesia. Journal of Experimental Psychology: Learning, Memory, and Cognition, 14, 758762.

Huppert, F. A., \& Piercy, M. (1979). Normal and abnormal forgetting in organic amnesia: Effects of locus on lesion. Cortex, 15, 385390.

Kaushall, P. I., Zetin, M., \& Squire, L. R. (1981). A psychosocial study of chronic, circumscribed amnesia. Journal of Nervous and Mental Disease, 169, 383-389.

Kovner, R., Mattis, S., \& Goldmeier, E. (1983). A technique for promoting robust free recall in chronic organic amnesia. Journal of Clinical Neuropsychology, 5, 65-71.

Mattis, S. (1976). Dementia rating scale. In R. Bellack, \& B. Karasu (eds.), Geriatric psychiatry (pp. 77-121), New York: Grune \& Stratton.
Mayes, A. R., Meudell, P. R., \& Neary, D. (1980). Do amnesics adopt inefficient strategies with faces and random shapes? Neuropsychologia, 18, 527-540.

Meudell, P. R., \& Mayes, A. R. (1982). Normal and abnormal forgetting: Some comments on the human amnesic syndrome. In L. A. Ellis (Ed.), Normality and pathology in cognition function (pp. 203-237). London: Academic Press.

Meudell, P. R., \& Mayes, A. R. (1984). Patterns of confidence loss in the cued recall of normal people with attenuated recognition memory: Their relevance to a similar amnesic phenomenon. Neuropsychologia, 22, 41-54.

Milner, B., Corkin, S., \& Teuber, H.-L. (1968). Further analysis of the hippocampal amnesic syndrome: 14-year follow-up study of H.M. Neuropsychologia, 6, 215-234.

Schacter, D. L. (1987). Implicit memory: History and current status. Journal of Experimental Psychology: Learning, Memory, and Cognition, 13, 501-518.

Shimamura, A. P. (1986). Priming effects in amnesia: Evidence for a dissociable memory function. Quarterly Journal of Experimental Psychology, 38A, 619-644.

Shimamura, A. P. (in press-a). Disorders of memory: The cognitive science perspective. In F. Boller \& J. Grafman (Eds.), Handbook of Neuropsychology. Amsterdam: Elsevier.

Shimamura, A. P. (in press-b). Forms of memory: Issues and directions. In J. L. McGaugh, N. M. Weinberger, \& G. Lynch (Eds.), Brain organization and memory: Cells, systems, and circuits. New York: Oxford University Press.

Shimamura, A. P., Jernigan, T. L., \& Squire, L. R. (in press). Korsakoff's syndrome: Radiological (CT) findings and neuropsychological correlates. Journal of Neuroscience.

Shimamura, A. P., \& Squire, L. R. (1984). Paired-associate learning and priming effects in amnesia: A neuropsychological study. Journal of Experimental Psychology: General, 113, 556-570.

Shimamura, A. P., \& Squire, L. R. (1986). Metamemory and memory: A study of the feeling-of-knowing phenomenon in amnesic patients. Journal of Experimental Psychology: Learning, Memory, and Cognition, 12, 452-460.

Shimamura, A. P., \& Squire, L. R. (1987). A neuropsychological study of fact memory and source amnesia. Journal of Experimental Psychology: Learning, Memory, and Cognition, 13, 464-473.

Squire, L. R. (1987). Memory and brain. New York: Oxford University Press.

Squire, L. R., \& Shimamura, A. P. (1986). Characterizing amnesic patients for neurobehavioral study. Behavioral Neuroscience, 100 , 866-877.

Squire, L. R., \& Zouzounis, J. A. (in press). Self-ratings of memory dysfunction: Different findings in depression and amnesia. Journal of Clinical and Experimental Neuropsychology.

Teuber, H. L., Milner, B., \& Vaughan, H. G. (1968). Persistent anterograde amnesia after stab wound of the basal brain. Neuropsychologia, 6, 267-282.

Weiskrantz, L. (1987). Neuroanatomy of memory and amnesia: A case for multiple memory systems. Human Neurobiology, 6, 93105.

Zola-Morgan, S., Squire, L. R., \& Amaral, D. G. (1986). Human amnesia and the medial temporal region: Enduring memory impairment following a bilateral lesion limited to field CA1 of the hippocampus. Journal of Neuroscience, 6, 2950-2967.

Received June 18, 1987

Revision received December 4, 1987 Accepted December 4, 1987 